\title{
Algebraic Probability Spaces
}

In this chapter we recall some definitions of quantum probability theory in the general framework developed by Accardi and several co-workers. Several expository papers in the scries Quantum Probability and Related Topics and two monographes $[68],[74]$ are already available in the literature. We will only illustrate the definitions by two examples which are included only with the aim to give a flavour of the relation with classical probability.

The reader is supposed to be familiar with the basic language and facts on $C^{*}$-algebras and von Neumann algebras. In Section 5 we recall only some fundamental definitions and results.

\subsection{Fundamental definitions}

Definition 1.1 $A$ *-algebra is a complex algebra $\mathcal{A}$ with an involution, denoted by $*$, with the following properties:

$$
\begin{aligned}
(\lambda a+\mu b)^{*} & =\bar{\lambda} a^{*}+\bar{\mu} b^{*} \\
\left(a^{*}\right)^{*} & =a \\
(a b)^{*} & =b^{*} a^{*}
\end{aligned}
$$

for all $a, b \in \mathcal{A}, \lambda, \mu \in \mathbb{C}$. An element $a$ of $\mathcal{A}$ is called positive if there exists $b \in \mathcal{A}$ such that $a=b^{*} b$.

We shall consider oftcn *-algebras with a unit denoted by $\mathbb{1}$.

Definition 1.2 Let $\mathcal{A}$ be a $*$-algebra with a unit $\mathbb{1}$. A state $\varphi$ on $\mathcal{A}$ is a linear map

$$
\varphi: \mathcal{A} \rightarrow \mathbb{C}
$$

with the properties: 
1. (positivity) $\varphi\left(a^{*} a\right) \geq 0$, for all $a \in \mathcal{A}$,

2. $($ normalisation $) \varphi(\mathbb{1})=1$.

We can now introduce the notion of algebraic probability space and algebraic random variable according to Accardi, Frigerio and Lewis [5].

Definition 1.3 An algebraic probability space is a pair $(\mathcal{A}, \varphi)$ where $\mathcal{A}$ is a *-algebra with unit and $\varphi$ is a state on $\mathcal{A}$.

Definition 1.4 Let $(\mathcal{A}, \varphi)$ be an algebraic probability space and let $\mathcal{B}$ be a $*$ algebra. An algebraic random variable on $\mathcal{A}$ with values in $\mathcal{B}$ is a $*$-homomorphism

$$
j: \mathcal{B} \rightarrow \mathcal{A} .
$$

The above quite general definitions will be always applied in "concrete" cases when $\mathcal{A}$ and $\mathcal{B}$ are at least $C^{*}$-algebras. In this case it is well known (see [21] Th. 2.1.10 p.60) that $\mathcal{A}$ and $\mathcal{B}$ are isomorphic to a norm-closed *-algebra of bounded operators in a Hilbert space.

\subsection{Classical probability spaces as algebraic}

A measurable space $(\Omega, \mathcal{F})$ clearly determines uniquely the commutative *algebra $\mathcal{A}=\mathcal{L}^{\infty}(\Omega, \mathcal{F} ; \mathbb{C})$ of $\mathcal{F}$-measurable, bounded, complex-valued functions on $\Omega$. A probability measure $\mathbb{P}$ on $\mathcal{F}$ induces a state $\varphi$ on $\mathcal{A}$ by

$$
\varphi(f)=\int_{\Omega} f(\omega) d \mathbb{P}(\omega) .
$$

Therefore the classical probability space $(\Omega, \mathcal{F}, \mathbb{P})$ can be considered also as the algebraic probability space $(\mathcal{A}, \varphi)$.

Let $(E, \mathcal{E})$ be a measurable space. Classical random variables on $\Omega$ with values in $E$ can be also interpreted as algebraic random variables. In fact, consider the $*$-algebra $\mathcal{B}=\mathcal{L}^{\infty}(E, \mathcal{E} ; \mathbb{C})$. A classical random variable $x$ can be described as an algebraic random variable by the $*$-homomorphism

$$
j: \mathcal{B} \mapsto \mathcal{A}, \quad j(f)=f \circ x .
$$

It is worth noticing here that each event can be represented by a projection in the $*$-algebra $\mathcal{A}$ through the identification with its indicator function.

\subsection{Quantum probability spaces}

Algebraic probability spaces appear as the basic structure in mathematical models for quantum mechanics. The mathematical structure however is (or at least can be assumed to be) richer with some more analytical properties on the algebra $\mathcal{A}$ and state $\varphi$. In order to distinguish an important class of algebraic probability spaces we give the following: 
Definition 1.5 A quantum probability space is a pair $(\mathcal{A}, \varphi)$ where $\mathcal{A}$ is a von Neumann algebra and $\varphi$ a $\sigma$-weakly continuous state on $\mathcal{A}$. $\mathcal{A}$.

An event in the quantum probability space $(\mathcal{A}, \varphi)$ is a projection operator in

$A$ quantum random variable in $(\mathcal{A}, \varphi)$ with values in a von Neumann algebra $\mathcal{B}$ is a $\sigma$-weakly continuous homomorphism

$$
j: \mathcal{B} \rightarrow \mathcal{A} .
$$

Although one could define events in the same way also when $\mathcal{A}$ is only a $C^{*}$-algebra the set of events in this case might be very poor. Indeed, if $\mathcal{A}$ is the $C^{*}$-algebra of complex-valued continuous functions on $\mathbb{R}^{d}$, then the set of events is trivial. On the other hand a von Neumann algebra $\mathcal{A}$ is generated by projections in $\mathcal{A}$.

Notice that, contrary to the classical case, the intersection (product) of two erents is no longer an event if the corresponding orthogonal projections do not commute.

We will show now in which sense a self-adjoint operator $X$ affiliated with the von Neumann algebra $\mathcal{A}$ of a quantum probability space $(\mathcal{A}, \varphi)$ can be considered both as a classical and a quantum random variable.

As we already noted we can assume that $\mathcal{A}$ is a sub von Neumann algebra of the von Neumann algebra $\mathcal{B}(h)$ of bounded operators on a Hilbert space $h$. Therefore $X$ is a self-adjoint operator on $h$.

Let $\mathcal{B}$ be the von Neumann algebra $L^{\infty}\left(\mathbb{R} ; \mathbb{C}^{\prime}\right)$. By the spectral theorem in functional calculus form ([78] Th. VIII.5 p. 262), for all $f \in \mathcal{B}$, we can define the element $f(X)$ of $\mathcal{A}$. The map

$$
j: \mathcal{B} \mapsto \mathcal{A}, \quad j(f)=f(X)
$$

is a ${ }^{*}$-homomorphism. This is clearly $\sigma$-weakly continuous because, for every increasing net $\left(f_{\alpha}\right)_{\alpha}$ of positive clements of $\mathcal{B}$ with least upper bound $f$ in $\mathcal{B}$ we have

$$
\sup _{\alpha} j\left(f_{\alpha}\right)=\sup _{\alpha} f_{\alpha}(X)=f(X)=j(f) .
$$

Therefore $X$ defines a quantum random variable.

Let $\Omega=\mathbb{R}$ and let $\mathcal{F}$ be the Borel $\sigma$-field on $\Omega$. Since both $j$ and $\varphi$ are $\sigma$-weakly continuous we can define a probability measure on $\mathcal{F}$ by putting

$$
\mathbb{P}(B)=\varphi\left(1_{B}(X)\right)
$$

where $1_{B}$ denotes the indicator function of a $B \in \mathcal{F}$. Thus we have constructed a classical probability space $(\Omega, \mathcal{F}, \mathbb{P})$.

We want to show now that $X$ can be represented as a classical real random variable on $(\Omega, \mathcal{F}, \mathbb{P})$. Fix a unit vector $e$ in $h$ and consider the closed subspace $h_{0}$ of $h$ generated by $e$ and vectors of the form

$$
f_{1}(X) f_{2}(X) \ldots f_{n}(X) e
$$


with $n \geq 1, f_{1}, \ldots, f_{n} \in \mathcal{B}$. Let $U$ be the unique unitary operator

$$
U: h_{0} \rightarrow L^{2}(\Omega, \mathcal{F}, \mathbb{P})
$$

such that

$$
U e=1, \quad U f_{1}(X) f_{2}(X) \ldots f_{n}(X) e=f_{1} f_{2} \ldots f_{n} .
$$

The operator $U$ is unitary because of the relation

$$
\int_{\mathbb{R}} g(\omega) d \mathbb{P}(\omega)=\varphi(g(X))
$$

for $g$ integrable with respect to $\mathbb{P}$ which follows immediately from (1.1).

It is easily checked that the following diagram commutes:

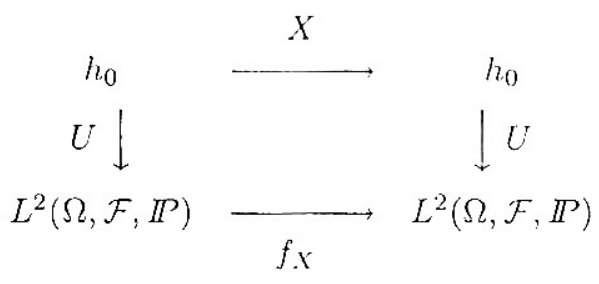

(where $f_{X}(x)=x$ ). Precisely we can show that

$-v \in D(X)$ if and only if $f_{X}(\cdot)(U v)(\cdot) \in L^{2}\left(\Omega, \mathcal{F}, l^{P}\right)$,

- if $w \in U(D(X))$, then $\left(U X U^{*} w\right)(\cdot)=f_{X}(\cdot) w(\cdot)$.

Therefore $U X U^{*}$ acts on $L^{2}(\Omega, \mathcal{F}, \mathbb{P})$ as the multiplication operator by a real function $f_{X}$ and the self-adjoint operator $X$ defines a classical real random variable.

Two non-commuting self-adjoint operators, however, cannot be represented as multiplication operators on the same Hilbert space $L^{2}(\Omega, \mathcal{F}, \mathbb{I P})$. This, roughly speaking, can be summarized by saying that $(\mathcal{A} . \hat{\varphi})$ is a quantum probability space "containing infinitely many classical probability spaces."

The following are concrete example in which the above fact occurs. We will use these models to illustrate also how classical probabilistic notions appear naturally in the quantum context.

Example 1.1 Spin matrices

Let $h=\mathscr{C}^{2}$, and let $\mathcal{A}$ be the von Neumann algebra of $2 \times 2$ complex valued matrices and $\varphi$ be any state on $\mathcal{A}$. The pair $(\mathcal{A}, \varphi)$ is clearly a quantum probability space. The self-adjoint operators on $h$ (also called spin matrices or Pauli matrices).

$$
\sigma_{1}=\left(\begin{array}{cc}
0 & 1 \\
1 & 0
\end{array}\right), \quad \sigma_{2}=\left(\begin{array}{cc}
0 & -i \\
i & 0
\end{array}\right), \quad \sigma_{3}=\left(\begin{array}{cc}
1 & 0 \\
0 & -1
\end{array}\right),
$$

represent three non-commuting quantum random variables. The above discussion shows immediately that $\sigma_{3}$ can be represented as a classical real random variable $x$ taking values $\{-1,+1\}$ with probabilities

$$
\mathbb{P}\{X=1\}=\varphi\left(\left(\begin{array}{ll}
1 & 0 \\
0 & 0
\end{array}\right)\right), \quad \mathbb{P}\{X=-1\}=\varphi\left(\left(\begin{array}{ll}
0 & 0 \\
0 & 1
\end{array}\right)\right) .
$$


Notice that -1 and 1 are the eigenvalues of $\sigma_{3}$ and the events $\left\{\sigma_{3}=1\right\}$ and $\left\{\sigma_{3}=-1\right\}$ are represented respectively by the orthogonal projections

$$
\left(\begin{array}{ll}
1 & 0 \\
0 & 0
\end{array}\right), \quad\left(\begin{array}{ll}
0 & 0 \\
0 & 1
\end{array}\right)
$$

In a similar way the laws of $\sigma_{1}$ and $\sigma_{2}$ can be computed using the spectral decompositions

$$
\begin{aligned}
& \sigma_{1}=\left(\begin{array}{cc}
1 / 2 & 1 / 2 \\
1 / 2 & 1 / 2
\end{array}\right)-\left(\begin{array}{cc}
1 / 2 & -1 / 2 \\
-1 / 2 & 1 / 2
\end{array}\right) \\
& \sigma_{2}=\left(\begin{array}{cc}
1 / 2 & -i / 2 \\
i / 2 & 1 / 2
\end{array}\right)-\left(\begin{array}{cc}
1 / 2 & i / 2 \\
-i / 2 & 1 / 2
\end{array}\right)
\end{aligned}
$$

Thus $\sigma_{1}, \sigma_{2}, \sigma_{3}$ are three non-commutative random variables with values in $\{-1 ;+1\}$.

Example 1.2 The harmonic oscillator

Let $h$ be the Hilbert space

$$
h=l^{2}(\mathbb{N}), \quad \mathbb{N}=\{0,1,2, \ldots\}
$$

with the canonical orthonormal basis $\left(e_{n}\right)$. We consider then the following operators:

1. annihilation operator

$$
\begin{aligned}
D(a) & =\left\{x=\left.\left(x_{n}\right)_{n} \in h\left|\sum_{n} n\right| x_{n}\right|^{2}<\infty\right\} \\
a e_{n} & =\sqrt{n} e_{n-1} \quad \text { if } n>0, \quad a e_{0}=0,
\end{aligned}
$$

2. creation operator

$$
\begin{aligned}
D\left(a^{*}\right) & =\left\{x=\left.\left(x_{n}\right)_{n} \in h\left|\sum_{n} n\right| x_{n}\right|^{2}<\infty\right\} \\
a^{*} e_{n} & =\sqrt{n+1} e_{n+1} \quad \text { for all } n \geq 0,
\end{aligned}
$$

3. number operator

$$
\begin{gathered}
D(N)=\left\{x=\left.\left(x_{n}\right)_{n} \in h\left|\sum_{n} n^{2}\right| x_{n}\right|^{2}<\infty\right\} \\
N e_{n}=a^{*} a e_{n}=n e_{n} \quad \text { for all } n \geq 0
\end{gathered}
$$

4. momentum (or electric field) operator

$$
D(p)=D(a)=D\left(a^{*}\right), \quad p=\frac{i}{\sqrt{2}}\left(a^{*}-a\right)
$$


5. position (or magnetic field) operator

$$
D(q)=D(a)=D\left(a^{*}\right), \quad q=\frac{1}{\sqrt{2}}\left(a^{*}+a\right) .
$$

It is well-known that the operators $N, p, q$ are self-adjoint. Therefore they can be considered as algebraic random variables. The canonical commutation relations (domains of the operators involved will be made precise later)

$$
\left[a, a^{*}\right]=a a^{*}-a^{*} a=\mathbb{1}, \quad(N+1) a=a N, \quad a^{*}(N+1)=N a^{*}
$$

$([, \cdot]$ denoting the commutator $)$ yield

$$
[q, p]=i \mathbb{1}, \quad[N, p]=i q, \quad[N, q]=\cdots i p .
$$

Therefore $N, p, q$ can not be represented as random variables on the same classical probability space.

The above model is called the Heisenberg representation of the canonical commutation relations over $\mathscr{C}$. It is well-known that it is unitarily equivalent to the Schrödinger representation. Indeed, letting $\left(H_{n}\right)_{n \geq 0}$ be the orthonormal secpen. of the Hermite polynomicls in $L^{2}\left(\mathbb{R} ; \pi^{-1 / 4} \exp \left(-x^{2} / 2\right) d x\right)$ the unitas operator

$$
\mathcal{U}: l^{2}(\mathbb{N}) \rightarrow L^{2}\left(\mathbb{R} ; \pi^{-1 / 4} \exp \left(-x^{2} / 2\right) d x\right), \quad \mathcal{U} e_{n}=H_{n}
$$

allows us to move from one representation to the other.

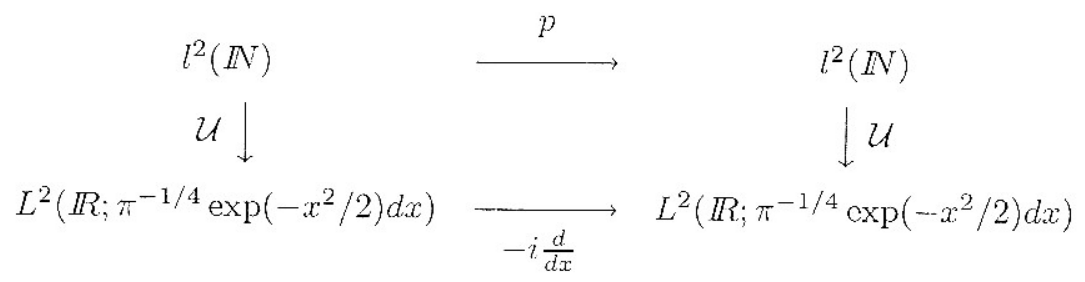

We refer to Meyer's book [68] for more details on this subject. The following is a "conversion table"

Heisenberg representation Schroedinger representation

$$
\begin{array}{cc}
p & -i \frac{d}{d x} \\
q & x \\
N & \frac{1}{2}\left(-\frac{d^{2}}{d x^{2}}+x^{2}-1\right)
\end{array}
$$

Let $\varphi$ be the state on $\mathcal{B}\left(L^{2}\left(\mathbb{R} ; \pi^{-1 / 4} \exp \left(-x^{2} / 2\right) d x\right)\right)$ defined by the unit vector $H_{0}$ (coinciding with the constant function 1 )

$$
\varphi(a)=\left\langle H_{0}, a H_{0}\right\rangle .
$$


Well-known facts allow us to compute easily the law of the random variables $p, q$. Notice first that the unitary groups generated by $q$ and $p$ are

$$
(\exp (i t q) v)(x)=\exp (i t x) v(x), \quad(\exp (i t p)) v(x)=v(x+t) .
$$

Therefore the characteristic functions of $p$ and $q$ in the state $\varphi$ are given by

$$
\begin{aligned}
& \varphi(\exp (i t q))=\left\langle H_{0}, \exp (i t q) H_{0}\right\rangle=\exp \left(-t^{2} / 4\right), \\
& \varphi(\exp (i t p))=\left\langle H_{0}, \exp (i t p) H_{0}\right\rangle=\exp \left(-t^{2} / 4\right) .
\end{aligned}
$$

Thus $p$ and $q$ are two non-commuting random variables with gaussian distribution with mean 0 and variance $1 / 2$.

Moreover we have

$$
\varphi(N)=\left\langle H_{0}, N H_{0}\right\rangle=\left\langle e_{0}, a^{*} a e_{0}\right\rangle=0 .
$$

Therefore, since $N$ is a non negative random variable, it is almost surely 0 with respect to the probability law determined by the state $\varphi$.

\subsection{Conditional expectation}

Examples given in the previous section motivate the following

Definition 1.6 Let $(\mathcal{A}, \varphi)$ be an algebraic probability space. For all element a of $\mathcal{A}$ we call the expectation, or mean value, of a in the state $\varphi$ the number $\varphi(a)$.

For all $a, b \in \mathcal{A}$ we define the covariance

$$
\varphi\left((a-\varphi(a))^{*}(b-\varphi(b))\right)
$$

and the variance

$$
\varphi\left((a-\dot{\varphi}(a))^{*}(a-\varphi(a))\right) .
$$

Notice that here we defined an algebraic analogue of expectation, covariance and variance essentially for bounded random variables. In fact $a$ is an element of $\mathcal{A}$.

We are now in a position to introduce the notion of conditional expectation.

Definition 1.7 Let $(\mathcal{A}, \varphi)$ be an algebraic probability space and let $\mathcal{A}_{0}$ be a sub *-algebra of $\mathcal{A}$ with unit $\mathbb{1}$. A conditional expectation is a linear map

$$
\mathbb{E}\left[\cdot \mid \mathcal{A}_{0}\right]: \mathcal{A} \mapsto \mathcal{A}_{0}
$$

with the following properties:

1. for all $a \in \mathcal{A}$ such that $a \geq 0$, we have $\mathbb{E}\left[a \mid \mathcal{A}_{0}\right] \geq 0$,

2. $\mathbb{E}\left[\mathbb{1} \mid \mathcal{A}_{0}\right]=\mathbb{1}$, 
3. for all $a_{0} \in \mathcal{A}_{0}$ and ail $a \in \mathcal{A}$ we have

$$
\mathbb{E}\left[a_{0} a ! \mathcal{A}_{0}\right]=a_{0} \mathbb{E}\left[a \mid \mathcal{H}_{0}:\right.
$$

4. fo: all $a \in \mathcal{A}$ we hace $\mathbb{E}\left[a^{*} \mid \mathcal{A}_{0}\right]=\left(\mathbb{E}\left[a \mid \mathcal{A}_{0}\right]\right)^{\prime}$,

5. for all $a \in \mathcal{A}_{0}$ we have

$$
\varphi(a)=\varphi\left(\mathbb{E}\left[a: \mathcal{A}_{0}\right]\right) .
$$

Example 1.3 The conditional expectations we shall use are of the following f.m. Let $h, h_{1}$ be complex separable Hilbert spaces. Consider the Hilbert space $k=h \otimes h_{1}$ and consider the $*$-algebras

$$
\mathcal{A}=\mathcal{B}(k), \quad \mathcal{A}_{0}=\mathcal{B}(h) \ldots \mathbb{1} .
$$

For all states $\varphi$ on $\mathcal{B}(h)$ and $\varphi_{1}$ on $\mathcal{B}\left(h_{1}\right)$. consider the state $\ell$ on $\mathcal{A}$ defined by

$$
\psi\left(a \otimes a_{1}\right)=\varphi(a) \varphi_{1}\left(a_{1}\right)
$$

for all $a \in \mathcal{B}(h), a_{1} \in \mathcal{B}\left(h_{1}\right)$. Then the linear map

$$
\mathbb{E}\left[\cdot \mid \mathcal{A}_{0}\right]: \mathcal{A} \mapsto \mathcal{A}_{0} . \quad \mathbb{E}\left[a \propto a_{1} \mid \mathcal{A}_{0}\right]=\varphi_{1}\left(a_{1}\right)(a \propto \mathbb{1})
$$

is a conditimal expectation.

Although our definition of conditional expectation in the $*$-algebraic language seenis to be quite natural, it is too restrictive in quantum probability. In fact, contrary to the classical case. given a quantum probability space $(\mathcal{A}, \varphi)$ and a sul, *-algebra let $\mathcal{A}_{0}$ of $\mathcal{A}$ with identity 11 , a conditional expectation $\mathbb{E}\left[\cdot \mid \mathcal{A}_{0}\right]: \mathcal{A} \rightarrow \mathcal{A}_{0}$ may not exists. A detailed discussion on conditional expectations in von Neumann algebras can be found in [72] and [77] by D. Pet\%.

Definition 1.8 Let $(\mathcal{A}, \varphi)$ be an algebraic probability space. A filtration is a family $\left(\left(\mathcal{A}_{\mathrm{j}}\right)_{t \geq 0}\right.$ of sub-*-alyebras of $\mathcal{A}$ such that

$$
\mathcal{A}_{s]} \subseteq \mathcal{A}_{l]}
$$

for all $s \leq t$.

Definition 1.9 Let $(\mathcal{A}, \varphi)$ be a quantum probability space and let $\left(\left(\mathcal{A}_{t]}\right)_{t \geq 0}\right.$ be a filtration. Suppose that, for all $t \geq 0$, there exists conditional expectations

$$
\mathbb{E}\left[\cdot \mid \mathcal{A}_{t]}\right]: \mathcal{A} \rightarrow \mathcal{A}_{t]} .
$$

The family $\left(\mathbb{E}\left[\cdot \mid \mathcal{A}_{t]}\right]\right)_{t \geq 0}$ is called projective if, for all $s \leq t$, we have

$$
\mathbb{E}\left[\mathbb{E}\left[\cdot \mid \mathcal{A}_{s]}\right] \mid \mathcal{A}_{t]}\right]=\mathbb{E}\left[\cdot \mid \mathcal{A}_{s\}}\right]
$$

We refer to the recent books of Meyer [68], Parthasarathy [74] and the references therein for a more detailed introduction to the language of quantum probability and other interesting examples. 


\subsection{Topologies on algebras}

A von Neumann algebra (or $W^{*}$-algebra) could be defined intrinsically as a special $C^{*}$-algebra. However we will consider only "concrete" von Neumann algebras, that is sub- $*$-algebras of operators on some Hilbert space $h$ closed under the weak or $\sigma$-weak or strong operator topology (see [21] pp.71-72).

These topologies can be defined through the convergence of nets.

Definition 1.10 Let $\left(x_{\alpha}\right)_{\alpha}$ be a net in $\mathcal{B}(h)$ and let $x \in \mathcal{B}(h)$. We say that:

1. $\left(x_{\alpha}\right)_{\alpha}$ converges weakly to $x$ if $\left\langle v, x_{\alpha} u\right\rangle$ converges to $\langle v, x u\rangle$ for every $v, u \in h$.

2. $\left(x_{\alpha}\right)_{\alpha}$ converges $\sigma$-weakly to $x$ if the sum $\sum_{n}\left\langle v_{n}, x_{\alpha} u_{n}\right\rangle$ converges to the sum. $\sum_{n}\left\langle v_{n}, x u_{n}\right\rangle$ for every pair of sequences $\left(v_{n}\right)_{n},\left(u_{n}\right)_{n}$ of elements of $h$ such that the series $\sum_{n}\left\|u_{n}\right\|^{2}$ and $\sum_{n}\left\|v_{n}\right\|^{2}$ converge.

3. $\left(x_{\alpha}\right)_{\alpha}$ converges strongly to $x$ if $x_{\alpha} u$ converges to $x u$ for every $u \in h$.

4. $\left(x_{\alpha}\right)_{\alpha}$ converges $\sigma$-strongly to $x$ if the sum $\sum_{n}\left\|\left(x_{\alpha}-x\right) u_{n}\right\|^{2}$ converges to 0 for every sequence $\left(u_{n}\right)_{n}$ of elements of $h$ such that the series $\sum_{n}\left\|u_{n}\right\|^{2}$ converges.

Clearly $\left(x_{\alpha}\right)_{\alpha}$ converges $\sigma$-weakly to $x$ if and only if, for every trace class operator $\rho$ in $h, \operatorname{tr}\left(x_{\alpha} \rho\right)$ converges to $\operatorname{tr}(x \rho)$.

The following facts will be frequently used:

(a) the $\sigma$-weak topology is stronger than the weak topology and not comparable to the strong one,

(b) the weak and $\sigma$-weak topology coincide on bounded subsets of $\mathcal{B}(h)$.

Definition 1.11 A von Neumann algebra is a ${ }^{*}$-subalgebra of $\mathcal{B}(h)$ containing Il closed in the weak (or by the bicommutant theorem [21] Th. 2.4.11 p.72 $\sigma$-weak or strong or $\sigma$-strong) topology.

We recall the following property of the cone of positive elements $\mathcal{A}_{+}$of a von Neumann algebra $\mathcal{A}$ (see [21] Lemma 2.4 .19 p.76).

Proposition 1.12 Let $\mathcal{A}$ be a von Neumann algebra of operators acting on a Hilbert space $h$ and let $\left(x_{\alpha}\right)$ be an increasing net in $\mathcal{A}_{+}$with an upper bound in $\mathcal{A}_{+}$. Then $\left(x_{\alpha}\right)_{\alpha}$ has a least upper bound $x=\sup _{\alpha} x_{\alpha}$ in $\mathcal{A}_{+}$and the net converges $\sigma$-strongly to $x$.

We define a useful class of positive functionals on a von Neumann algebra.

Definition 1.13 Let $\mathcal{A}$ be a von Neumann algebra of operators acting on the Hilbert space $h$ and let $\omega$ be a positive linear functional on $\mathcal{A}$. We say that $\omega$ is normal if

$$
\sup _{\alpha} \omega\left(x_{\alpha}\right)=\omega\left(\sup _{\alpha} x_{\alpha}\right) .
$$


The following fundamental properties of states on a von Neumann algebra are well-known (see [21] Theorem 2.4.21 p.76).

Theorem 1.14 Let $\mathcal{A}$ be a von Neumann algebra of operators acting on a Hilbert space $h$ and let w be a state on $\mathcal{A}$. The following conditions are equindlent:

1. $\omega$ is normal,

2. $\omega$ is $\sigma$-weakly continuous.

3. there exists a density matrix $\rho$ (i.e. a positiv trace-class operator on $h$ with $\operatorname{tr}(\rho)=1$ ) suct that $\omega(x)=\operatorname{tr}(\rho x)$.

We shall use often a consequence of the equiralence of 1 and 2. Let us recall that a subset $\mathcal{E}$ of a Hilbert space $h$ is called total in $i$ if the linear manifold generated by $\mathcal{E}$ is dense in $h$.

Proposition 1.15 Let $\mathcal{A}$ and $\mathcal{B}$ be von Neumann algebras, $\mathcal{B}$ acting on a Hilbert space $h$, and let $T: \mathcal{A} \rightarrow \mathcal{B}$ be a positive linear map. The following conditions are equivalent:

1. T is r-wcakly continuous (i.c. continuoun with respect to the o-ukcuti topologies on $\mathcal{A}$ and $\mathcal{B}$ ).

2. for every increasing net $\left(x_{\alpha}\right)_{\alpha}$ in $\mathcal{A}_{+}$with least upper bound $x$ in $\mathcal{A}_{+}$the: increasing net $\left(T x_{\alpha}\right)_{\alpha}$ in $\mathcal{B}_{+}$conceryes $\sigma$-weakly to $T x$,

3. for every increasing net $\left(x_{\alpha}\right)_{a}$ in $\mathcal{A}_{+}$with least upper bound $x$ in $\mathcal{A}_{+}$we have

$$
\lim _{\alpha}\left\langle u,\left(T x_{\alpha}\right) u\right\rangle=\sup _{\alpha}\left\langle u,\left(T x_{\alpha}\right) u\right\rangle=\langle u,(T x) u\rangle
$$

for each u in a linear submanifold of he which is norm-dense in h,

$\therefore$ for every increasing net $\left(._{\alpha}\right)_{a}$ in $\mathcal{A}_{+}$with least upper bound $x$ in $\mathcal{A}_{+}$we have

$$
\lim _{a}\left\langle v,\left(T x_{a}\right) u\right\rangle=\langle v,(T x) u\rangle
$$

for each $v, u$ in a total subset of h.

Proof. 1 implies 2. Indeed it suffices to note that the net $\left(x_{\alpha}\right)_{\alpha}$ converges weakly to $x$ by Proposition 1.12.

2 implies 3. Obvious since the linear functionals on $\mathcal{B} y \rightarrow\langle u, y u\rangle$ with $u \in h$ are $\sigma$-weakly continuous.

3 implies 4 . We show first that 3 implics that the not $\left(\left\langle u,\left(T x_{\alpha}\right) u\right\rangle\right)_{a}$ converges to $\langle u,(T x) u\rangle$ for each $u \in h$. Indeed. for every $\varepsilon>0$, there exists $u_{\xi}$ 
in the dense subset such that $\left\|u-u_{\varepsilon}\right\|<\varepsilon$. The inequality $T x_{\alpha} \leq T x$ clearly implies $\left\|T x_{\alpha}\right\| \leq\|T x\|$. We have then

$$
\begin{aligned}
\left|\left\langle u,\left(T x_{\alpha}\right) u\right\rangle-\langle u,(T x) u\rangle\right| & \leq\left|\left\langle u-u_{\varepsilon},\left(T x_{\alpha}\right) u\right\rangle-\left\langle u-u_{\varepsilon},(T x) u\right\rangle\right| \\
& +\left|\left\langle u_{\varepsilon},\left(T x_{\alpha}\right)\left(u-u_{\varepsilon}\right)\right\rangle-\left\langle u_{\varepsilon},(T x)\left(u-u_{\varepsilon}\right)\right\rangle\right| \\
& +\left|\left\langle u_{\varepsilon},\left(T x_{\alpha}\right) u_{\varepsilon}\right\rangle-\left\langle u_{\varepsilon},(T x) u_{\varepsilon}\right\rangle\right| \\
& \leq\left\|u-u_{\varepsilon}\right\|\left(\left\|T x_{\alpha}\right\|+\|T x\|\right)\left(\|u\|+\left\|u_{\varepsilon}\right\|\right) \\
& +\left|\left\langle u_{\varepsilon},\left(T x_{\alpha}\right) u_{\varepsilon}\right\rangle-\left\langle u_{\varepsilon},(T x) u_{\varepsilon}\right\rangle\right| .
\end{aligned}
$$

Therefore we have

$$
\lim _{\alpha}\left|\left\langle u,\left(T x_{\alpha}\right) u\right\rangle-\langle u,(T x) u\rangle\right| \leq 2 \varepsilon\|T x\|(2\|u\|+\varepsilon) .
$$

Since $\varepsilon$ is arbitrary, the net $\left(\left\langle u,\left(T x_{\alpha}\right) u\right\rangle\right)_{\alpha}$ converges to $\langle u,(T x) u\rangle$ for each $u \in h$.

By the polarisation identity

$$
\left\langle v,\left(T x_{\alpha}\right) u\right\rangle=\frac{1}{4} \sum_{k=0}^{3} i^{-k}\left\langle v+i^{k} u,\left(T x_{\alpha}\right)\left(v+i^{k} u\right)\right\rangle
$$

it follows then that (1.2) holds for each $v, u \in h$.

4 implies 3 . In fact 4 implies that (1.2) holds for each $v, u$ in the dense subset of $h$ linearly spanned by the total set. This linear span is obviously dense.

3 implies 2. Let $\left(v_{n}\right)_{n \geq 0},\left(u_{n}\right)_{n \geq 0}$ be two sequences of vectors in $h$ such that the sequences $\left(\left\|v_{n}\right\|\right)_{n \geq 0},\left(\left\|u_{n}\right\|\right)_{n \geq 0}$ are square-summable. We must show that

$$
\lim _{\alpha} \sum_{n \geq 0}\left\langle v_{n},\left(T x_{\alpha}\right) u_{n}\right\rangle=\sum_{n \geq 0}\left\langle v_{n},(T x) u_{n}\right\rangle .
$$

To this end, for every $\varepsilon>0$, take an integer $\nu$ such that

$$
\sum_{n>\nu}\left\|u_{n}\right\|^{2}<\varepsilon, \quad \sum_{n>\nu}\left\|v_{n}\right\|^{2}<\varepsilon .
$$

We have then

$$
\begin{aligned}
\left|\sum_{n \geq 0}\left\langle v_{n},\left(T x_{\alpha}\right) u_{n}\right\rangle-\sum_{n \geq 0}\left\langle v_{n},(T x) u_{n}\right\rangle\right| \\
\leq \quad\left(\left\|T x_{\alpha}\right\|+\|T x\|\right) \sum_{n>\nu}\left\|u_{n}\right\| \cdot\left\|v_{n}\right\| \\
+\left|\sum_{n=0}^{\nu}\left\langle v_{n},\left(T x_{\alpha}\right) u_{n}\right\rangle-\sum_{n=0}^{\nu}\left\langle v_{n},(T x) u_{n}\right\rangle\right| .
\end{aligned}
$$

The first term, since $\left\|T x_{\alpha}\right\| \leq\|T x\|$, can be estimated by

$$
\|T x\|\left(\sum_{n>\nu}\left\|u_{n}\right\|^{2}+\sum_{n>\nu}\left\|v_{n}\right\|^{2}\right)<2 \varepsilon\|T x\| .
$$


Moreover, as we have shown in the proof that 3 implies 4 , the property 3 implies the convergence of the net $\left(\left\langle v,\left(T x_{\alpha}\right) u\right\rangle\right)_{\alpha}$ to $\langle v,(T \dot{x}) u\rangle$ for each $v, u \in h$. We have then

$$
\lim _{\alpha}\left|\sum_{n \geq 0}\left\langle v_{n},\left(T x_{\alpha}\right) u_{n}\right\rangle-\sum_{n \geq 0}\left\langle v_{n},(T x) u_{n}\right\rangle\right| \leq 2 \varepsilon\|T x\| .
$$

Since $\varepsilon$ is arbitrary this show's that (1.2) holds.

2 implies 1 . Let $\left(x_{\alpha}\right)$ a be a net in $\mathcal{A}$ converging $\sigma$-weakly to $x$. For every pair $\left(v_{n}\right)_{n \geq 0},\left(u_{n}\right)_{n \geq 0}$ of sequences of vectors in $h$ such that $\left(\left\|v_{n}\right\|\right)_{n \geq 0},\left(\left\|u_{n}\right\|\right)_{n \geq 0}$ are square-summable let $\omega$ be the $\sigma$-weakly continuous functional on $\mathcal{B}$

$$
\omega(y)=\sum_{n \geq 0}\left\langle v_{n} \cdot y u_{n}\right\rangle .
$$

By the complex polarisation identity $\omega$ can be written as a lincar combination of four positive linear functionals $\omega_{k}, k=0,1,2,3$. with

$$
\omega_{k}(y)=\sum_{n \geq 0}\left\langle\left(v+i^{k} u\right) \cdot y\left(v+i^{k} u\right)\right\rangle .
$$

Therefore, in order to show that 2 implies 1 , it suffices to prove that the not $\left(\omega\left(T x_{\alpha}\right)\right)_{a}$ converges to $\omega(T x)$ for every positive linear functional $\omega$ of the above form.

If, for such an $\omega$, we have $\omega(T \mathbb{1})=0$, then we have also $\omega\left(T T^{\prime}\right)=0$ for each self-adjoint element $x$ of $\mathcal{A}$ because $-\|x\| \mathbb{1} \leq x \leq\|x\| \mathbb{1}$ and $T$ is positive. Therefore

$$
\omega(T \cdot x)=\omega\left(T\left(x+x^{*}\right)\right) / 2+i \omega\left(T(x-x)^{*} / i\right) / 2=0
$$

for each $x \in \mathcal{A}$. Thus there is nothing to prove.

If $\omega(T \mathbb{1})>0$, then, letting

$$
\tilde{\omega}(y)=\omega(T y) / \omega(T \mathbb{1}),
$$

we define a state $\tilde{\omega}$ on $\mathcal{A}$. Condition 2 implies then that $\tilde{\omega}$ is normal. Then, by virtue of Theorem 1.14 , it is $\sigma$-weakly continuous so that

$$
\lim _{\alpha} \omega\left(T x_{\alpha}\right)=\lim _{\alpha} \omega(T \mathbb{1}) \tilde{\omega}\left(T x_{\alpha}\right)=\omega(T \mathbb{1}) \tilde{\omega}(T x)=\omega(T x) .
$$

This completes the proof.

A map enjoying the property 3 is also called normal. We will often use the above equivalence to show that a positive map is continuous for the $\sigma$-weak topology. Moreover we will often use normal with the same meaning of $\sigma$-weak when no confusion can arise.

We refer to the book [21] for more detailed results on von Neumann algebras. 